\title{
Amine variations in faecal content in the first weeks of life of newborns in relation to breast-feeding or infant formulas
}

\author{
Lorena Suárez ${ }^{1,2,3 \dagger}$, María Moreno-Luque ${ }^{1} \dagger$, Irene Martínez-Ardines ${ }^{4}$, Nereida González ${ }^{1}$, Patricia Campo ${ }^{5}$, \\ Palmira Huerta-Cima ${ }^{6}$ and Manuel Sánchez ${ }^{1,2,3 *}$ \\ ${ }^{1}$ Farmacologí, Departamento de Medicina, Universidad de Oviedo, 33006 Oviedo, Spain \\ ${ }^{2}$ Instituto Universitario de Oncología del Principado de Asturias (IUOPA), 33006 Oviedo, Spain \\ ${ }^{3}$ Instituto de Investigación Sanitaria del Principado de Asturias (ISPA), 33011 Oviedo, Spain \\ ${ }^{4}$ Centro de Salud La Fresneda, Servicio de Salud del Principado de Asturias (SESPA), 33429 Siero, Spain \\ ${ }^{5}$ Centro de Salud El Parque-Somió (SESPA), 33203 Gijón, Spain \\ ${ }^{6}$ Servicio de Obstetricia, Hospital Universitario Central de Asturias, 33011 Oviedo, Spain \\ (Submitted 10 September 2018 - Final revision received 19 July 2019 - Accepted 31 July 2019)
}

\section{Abstract}

Breast-feeding is the ideal nutrition for a newborn's integral necessities. It seems crucial therefore to know its composition in order to provide suitable infant formula when required. Of these, polyamines (with lactation and the microbiota being its intestinal source) are involved in the development of gut epithelium and immunity. Safety concerns limit human intervention studies. Therefore, we studied the amounts of polyamines supplied by breast milk (varying among mothers) or infant formula feeding, up to day 30 postpartum, in the faeces of newborns. Independent samples (68) of breast milk from fifty-nine healthy Caucasian woman (day 0, 1, 3, 5, 7, 15 and 30 postpartum) who had natural deliveries after week 38, same-day faeces of newborns when available (eighty-one from breast milk and fifty-five from infant formula fed) and six infant formulas were collected and the polyamine content was determined by HPLC. In breast milk, polyamines and isoamylamine (a primary amine), with inter-individual variations, increased over time (with a higher content of spermidine; no other amines were present). Overall, they were much higher than in infant formula. By the 2nd week after birth, polyamines, cadaverine and tyramine, but not isoamylamine, were higher in the faeces of those fed infant formula compared with those fed breast milk. Cadaverine and tyramine could be used to predict the feeding type used for newborns. The differences in the content may be related to distinct colonisation of amine-producing bacteria, which can be established by polyamines. Further studies are required to determine the clinical utility of these findings.

Key words: Polyamines: Trace amines: Breast-feeding: Infant formulas: Faecal amines

Breast-feeding is a matter of interest as it has been associated with benefits on different aspects of human health. These cover a broad spectrum of immediate effects, such as the maturation of intestinal epithelium ${ }^{(1,2)}$ and the immune system in the newborns ${ }^{(3,4)}$, the decrease in mortality due to infectious diseases, especially in countries with lower income ${ }^{(5)}$. Longterm effects also include, among others, a lower probability of obesity in adults ${ }^{(6,7)}$, atopy, asthma and hypertension ${ }^{(8)}$, an increase in the intelligence quotient and cognition ${ }^{(9)}$ and a delay in senescence ${ }^{(10)}$. However, concerns exist about some of the long-term benefits of breast-feeding (which were based on observational studies that might have been overstated $\left.^{(11-15)}\right)$ as well as whether it should be maintained at all costs ${ }^{(16)}$.
During the first 6 months, breast-feeding is associated with health advantages compared with infant formulas, which have encouraged the promotion of breast-feeding as the 'gold standard' to cover the newborn's integral necessities ${ }^{(17,18)}$. This fact gives relevant search for crucial components to identify the proper alternative feeds in case breast-feeding is unfeasible. Of the multiple lines of interest, attention has been given to the role of polyamines contained in breast milk. These are ubiquitous polycationic compounds present in all living cells. In mammals, they are synthesised from ornithine being converted to putrescine, by means of ornithine decarboxylase, which is then enzymatically converted to spermidine and then to spermine. The dietary supply of polyamines is also important to maintain essential physiological functions ${ }^{(19)}$.

* Corresponding author: Dr M. Sánchez, fax +34 985103551, email sanchezf@uniovi.es

$\dagger$ These authors contributed equally to this work. 
Polyamines stimulate the growth and healing of the gastrointestinal mucosa in animal models ${ }^{(20-23)}$. When they were introduced to milk during lactation, the time of intestinal mucosa proliferation was shortened ${ }^{(24-27)}$, with an effect on the immune system $^{(28,29)}$, supporting their role in the maturation of the intestinal barrier during the neonatal period. Besides trophic effects, polyamines and biogenic primary amines present in the intestinal content modulate spontaneously initiated gastrointestinal motility in the smooth muscle/interstitial cells of Cajal/platelet-derived growth factor receptor-positive cells (SIP) syncytium ${ }^{(30)}$. This may facilitate gastric emptying and drive segmentation and peristalsis of the intestine ${ }^{(30,31)}$, having a complementary role in the digestion and absorption of nutrients in the microvilli. But neither the specific cellular location (SIP syncytium, enteric innervation and/or smooth muscle cells) for the functional effects of biogenic amines nor the specific chemosensory system receptors have yet been identified. However, non-olfactory trace amine-associated receptors are expressed in the intestine ${ }^{(32)}$.

Based on its composition, breast milk has been classified into three stages: colostrum, transitional and mature milk ${ }^{(33)}$, with variations in the concentration of polyamines ${ }^{(34)}$; all of which are higher than the content in infant formulas. A wide range of polyamine concentrations exists in human breast milk between different mothers depending on several factors, such as age, race, obesity ${ }^{(35)}$, type of nutrition ${ }^{(36,37)}$, mode of delivery $^{(37)}$, premature birth ${ }^{(34,36)}$ and mastitis ${ }^{(38)}$. In the newborns, besides oral intake, biliary and pancreatic exocrine secretion ${ }^{(39)}$, cell exfoliation and intestinal microbiota ${ }^{(40-42)}$ are additional intestinal sources of polyamines, which, in turn, may contribute to the establishment of the microbiota ${ }^{(43-45)}$. Absorption capability also determines the homeostasis of intestinal polyamines ${ }^{(46)}$, which is mainly carried out in the duodenum and ileum.

Despite the immediate known beneficial enteric effects of polyamines, their intestinal content and of other biogenic amines have not been determined in newborns to establish whether variations exist depending on oral ingestion, and this could be of importance regarding the effects described. Safety concerns limit experimental studies in human, leading us to study whether potential variations in polyamine intake (based on the content in human breast milk among mothers and in infant formulas) and/or on the type of feeding may determine the content in faeces of newborns as a function of time from childbirth to day 30 postpartum.

\section{Experimental methods}

\section{Study design and participants}

This was initially designed as a cohort study, from the first to the 30th day postpartum, to analyse the content of biogenic amines in the mother's breast milk (or infant formula) and in the faeces of her child. This was not feasible; thus, an observational study was carried out in independent samples comprising fifty-nine Caucasian women and their children. Of them, thirty-nine provided breast milk and twenty infant formula feeding. They were invited to participate consecutively, as they were giving birth at the Hospital Universitario Central de Asturias in Oviedo (Spain) or when attended by the midwife during visits to the health centre (Centro de Salud Parque-Somió, Gijón, Spain), in the weeks established to collect samples, as long as they fulfilled the inclusion criteria. It was not possible to obtain samples of both breast milk and faeces in all cases, since sometimes at the same time of day 1 of the samples was not available.

The proposal of the study was explained to the participants who were previously required to provide signed informed consent. The inclusion criteria for the mothers (giving breast milk or infant formula feeding but not mixed feeding at any time) were age from 20 to 40 years, natural vaginal childbirth, the absence of chronic diseases or any disease 2 weeks before the delivery, non-obese and of at least 38 weeks of pregnancy.

The mean age of the mothers supplying breast milk was 33.44 (sE 0.84 ) years old ( $n$ 39) and of those opting for infant formula was 31.08 (se 1.4 ) years old ( $n 20)$. The mean number of deliveries were, respectively, 1.48 (SE $0 \cdot 13$ ) and 1.38 (SE 0.18). All were vaginally delivered, with thirty-one $(52.5 \%)$ boys and twenty-eight (47.5\%) girls.

The study was approved by the Comité de Ética de la Investigación del Principado de Asturias, Spain (reference $24 / 2011$ ) and was conducted according to the guidelines of the Declaration of Helsinki.

\section{Samples of human breast milk, infant formulas and faeces of the newborn babies}

Samples of human milk were collected in sterile Eppendorf( tubes of $1.5 \mathrm{ml}$, with the following schedule: the same day of delivery (day of giving birth, considered day 0 ), the next day or day 1 , the $3 \mathrm{rd}, 5$ th, 7 th, 15 th and 30 th days after childbirth. The minimum volume considered to determine milk amines was $50 \mu \mathrm{l}$. The total samples were $n$ 68. The samples of breast milk were maintained at $4^{\circ} \mathrm{C}$ while transported to the laboratory and then were preserved at $-80^{\circ} \mathrm{C}$.

Women who chose infant formula, liquid or powder, were asked for a sample to analyse different brands ( $n$ 6).

The samples of newborn faeces (for breast milk $n 81$ and for infant formula fed $n$ 55) were collected following the same schedule and hours as breast milk samples. For this, the diapers were brought refrigerated to the laboratory, then a sample of faeces of approximately $150 \mathrm{mg}$ was introduced in sterile Eppendorf ${ }^{\odot}$ tubes in $10 \%$ glycerol to be preserved at $-80^{\circ} \mathrm{C}$, until they were used. Mother-infant pairs of breast milk-fed newborns were forty-one.

\section{Determination of amines in the different types of milk and newborns faeces by HPLC}

The amines were determined using a pre-column derivatisation method, as previously described ${ }^{(47,48)}$. The samples of faeces were homogenised and then centrifuged at $4000 \mathrm{rpm}$ for $3 \mathrm{~min}$ at room temperature to obtain $300 \mu$ l supernatant. These and the samples of milk were treated with perchloric acid to reach a final concentration of $15.8 \%\left(10 \mathrm{~min}\right.$ at $\left.4^{\circ} \mathrm{C}\right)$. The extracts were then centrifuged at $10000 \mathrm{~g}$ for $30 \mathrm{~min}$ at $4{ }^{\circ} \mathrm{C}$, and $0.2 \mathrm{ml}$ supernatants were collected and then neutralised with $0.3 \mathrm{ml}$ of a saturated solution of $\mathrm{NaHCO}_{3}$ and the standard ( $1.25 \mu \mathrm{l}$ or $5 \mu \mathrm{l}$ of $1 \mathrm{mmol}$ solution, respectively for milk or faeces). The samples were dansylated overnight ( $16 \mathrm{~h}$ ) with $0.5 \mathrm{ml}$ of a solution containing $5 \mathrm{mg} / \mathrm{ml}$ of dansyl chloride in acetone, 
then proline $100 \mathrm{mg} / \mathrm{ml}$ was added and left for $30 \mathrm{~min}$. After one extraction with toluene the organic phase was dried under a nitrogen atmosphere at $42^{\circ} \mathrm{C}$ and afterwards was resuspended in $0.2 \mathrm{ml}$ acetonitrile (VWR).

The samples were chromatographed in an HPLC (Shimadzu Prominence $)$ using a C18 $(2.5 \mu \mathrm{m}, 3.0 \times 75 \mathrm{~mm})$ reverse-phase column (XBridge from Waters), at room temperature $22-24^{\circ} \mathrm{C}$, and equipped with two LC-20AD pumps, a fluorescence detector (RF-20A), an SIL-20AD ${ }_{\text {HT }}$ auto sampler injector at $4^{\circ} \mathrm{C}$ and a DGU-20A 3 degasser. The binary gradient used a flow rate of $0.55 \mathrm{ml} / \mathrm{min}$ as follows: $0-0.5 \mathrm{~min}, 0 \% \mathrm{~B}$; 0.5-10 $\mathrm{min}, 75 \% \mathrm{~B}$; 10-11.30 min, 75\% B; 11.30-11.50 min, 0\% B, where solvent A was $45 \%$ of acetonitrile and solvent B was $100 \%$ of acetonitrile. The injection volume was of $5 \mu \mathrm{l}$.

The duration of the chromatogram was $14 \mathrm{~min}$. The identification of compounds was performed by means of their retention time (standard: $05.88 \mathrm{~h}$, isoamylamine: $07.04 \mathrm{~h}$, putrescine: $07.38 \mathrm{~h}$, cadaverine: $07.83 \mathrm{~h}$, tyramine: $09.89 \mathrm{~h}$, spermidine: $10.38 \mathrm{~h}$, spermine: $12.41 \mathrm{~h}$ ) and a wavelength of excitation of $365 \mathrm{~nm}$ and of emission of $510 \mathrm{~nm}$. All measurements were made using Shimadzu LCsolution Version 1.25 software. The data were the mean of duplicate injections. Quantification of biogenic amines was performed using 2-hydroxydiaminopropane as an internal standard, being expressed as $\mathrm{nmol} / \mathrm{ml}$ of breast milk or infant formula or in $\mathrm{nmol} / \mathrm{mg}$ of faeces.

\section{Chemicals}

The following drugs were used: putrescine (tetramethylenediamine), spermidine ( $\mathrm{N}$-(3-aminopropyl)-1,4-butanediamine), spermine (N,N'-bis(3-aminopropyl)-1,4-butanediamine), isoamylamine (isopentylamine: 1-amino-3-methylbutane), cadaverine (cadaverine dihydrochloride), tyramine (2-(4-hydroxyphenyl) ethylamine) tyramine hydrochloride (4-(2-aminoethyl) phenol hydrochloride) and 2-hydroxydiaminopropane were purchased from Sigma-Aldrich. The drugs were dissolved in purified water with a resistance of $10-15 \mathrm{M} \Omega \times \mathrm{cm}$.

\section{Statistical analysis}

The sample size was conditioned by the feasibility of recruitment, considering at least four different independent samples for each group of days $(n)$, taken as reference similar articles in this field ${ }^{(34)}$. This minimum size of sample corresponded in each case to an equal number of mother's breast milk, infant formulas or child's faeces.

The samples were also grouped as ordinal variables following the interval days of the three stages of breast milk: colostrum from days 1 to 5, transitional from days 6 to 14 and mature from days 15 to 30 postpartum. These intervals were also used to analyse the amine content in the faeces of breast-fed and infant formula-fed newborns.

Exploratory analysis was used to determine whether the distribution of amine values in each group of variables significantly differed from a normal distribution. Kolmogorov-Smirnov and Levene's test, respectively, were used for the analysis of normality and homoscedasticity of each group of variables, assuming normality if $P>0 \cdot 05$. Since for several groups this was violated, non-parametric statistics were used.
The concentrations of biogenic amines in breast milk and faeces were represented as box and whisker plots for the days of the collected sample. The statistical difference in two independent variables (non-parametric), such as for the differences in a particular amine between breast milk and infant formula, was calculated by means of the Mann-Whitney $U$ test. The difference in the amine values obtained between several independent groups of days in different types of feeding was calculated by means of the KruskalWallis test (pairwise comparison), followed by Mann-Whitney test adjusted by Bonferroni correction for multiple tests. The effect size ( $r$ ) of the comparison of groups was calculated by means of the following formula: $r \mathrm{Z}$ score $/ \sqrt{ } \mathrm{N}$. The group comparison may result in small $(0 \cdot 1)$, medium $(0 \cdot 3)$ or large $(0 \cdot 5)$ effect $^{(49)}$.

The mean ratios (and standard errors of the mean) of isoamylamine to putrescine, spermidine, spermine, cadaverine and tyramine were used to represent the data and fitted to an exponential equation to obtain the time constant of the decrease $(\tau)$.

The Spearman's rank correlation $\left(r_{s}\right)$ (two tailed) was used to determine the association between different amines contained in breast milk, infant formulas or child faeces as well as whether the content in the type of feeding is related to that determined in the faeces. Binary logistic regression was used to establish the interaction of biogenic amines in faeces as predictors of the type of newborn feeding. For all analyses, values of $P \leq 0.05$ were considered as significant. The analysis was performed by means of IBM SPSS Statistics version 24.0 (IBM Corp.).

\section{Results}

\section{Content of amines in human breast milk and infant formulas}

Data showed that varying concentrations of polyamines ton he day of giving birth, as demonstrated by the Kruskal-Wallis test $(H(2)=12.56, P=0.002, n 30)$; putrescine (Fig. 1(A)) is significantly lower than spermine (Fig. $1(\mathrm{C}))(z=-3 \cdot 5, P=0 \cdot 001$, $r-0 \cdot 8$ ) (Mann-Whitney tests, with adjusted $P$ values), but not lower than spermidine (Fig. 1(B)). No differences exist between spermidine (Fig. 1(B)) and spermine (Fig. 1(C)). The primary amine, isoamylamine (Fig. 1(D)), was present in breast milk at the highest concentration on the day of giving birth (KruskalWallis test $(H(3)=27 \cdot 06, P<0 \cdot 001, n 40)$. No other biogenic amines were detected. Pairwise comparisons (Mann-Whitney tests with adjusted $P$ values) showed that the concentration of putrescine (Fig. 1(A)) $(z=5.09, P<0.001, r 1.14)$ and spermidine (Fig. 1(B)) $(z=3.35, P=0.005, r 0.75)$ were lower than those of isoamylamine (Fig. 1(D)) (Supplementary Table S1).

Polyamines showed an increase during postpartum (from day of giving birth (referred as 0) to day 30) with significant differences in concentrations across the different groups of days, as revealed by the Kruskal-Wallis test: putrescine (Fig. $1(\mathrm{~A})) \quad(H(6)=15.49, \quad P=0.017, \quad n$ 66), spermidine (Fig. 1(B)) $(H(6)=42.94, P<0.001, n$ 69), spermine (Fig. 1(C)) $(H(6)=27 \cdot 87, P<0 \cdot 001, n 66)$. Isoamylamine (Fig. 1(D)) equally significantly increased $(H(6)=27.52, P<0 \cdot 001, n 68)$ over time. The comparison (Mann-Whitney tests, with adjusted $P$ values) of concentrations across the groups of days showed that putrescine (Fig. 1(A)) increased significantly when values of day 3 were 
(A)

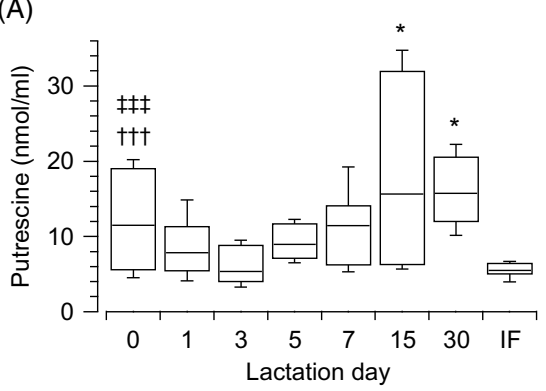

(C)

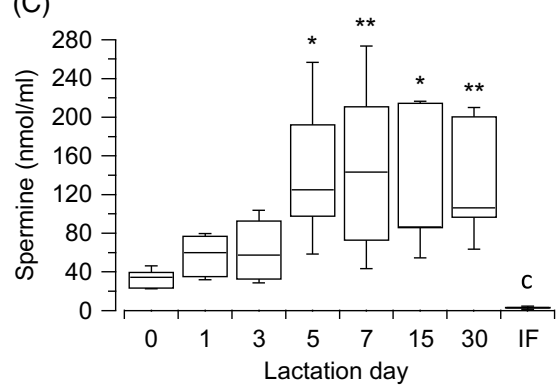

(B)

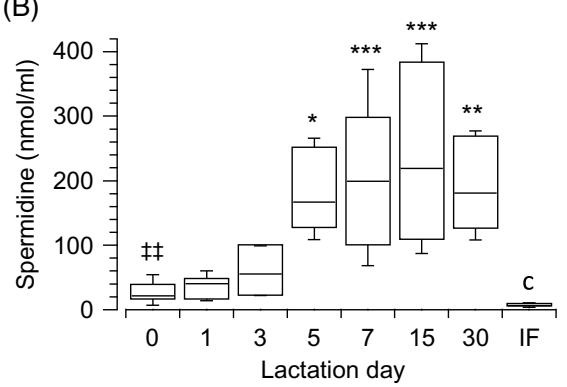

(D)

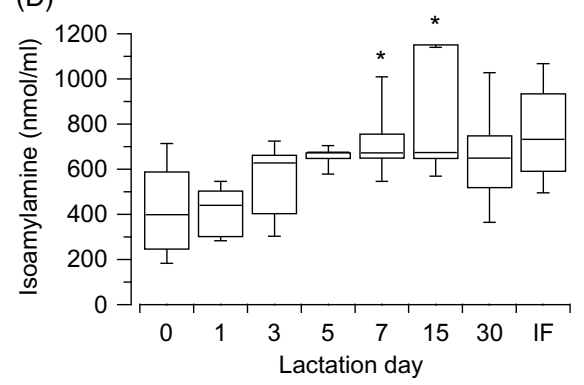

Fig. 1. Box and whisker plots of the values ( $\mathrm{nmol} / \mathrm{ml}$ ) of putrescine (A), spermidine (B), spermine (C) and isoamylamine (D) in the breast milk, from the day of giving birth (day 0 ) to day 30 postpartum. In (A) ${ }^{\star} P \leq 0.05$ in comparison with day 3 ; in (B) and $(\mathrm{C}){ }^{*} P \leq 0.05,{ }^{* *} P \leq 0.01$ and ${ }^{* * *} P \leq 0.001$ with respect to the day of giving birth, and in (D) ${ }^{*} P \leq 0.05$ day 7 with respect to day 0 and also day 15 with respect to day 1 . $\dagger \dagger \dagger P \leq 0.001$ with respect to spermine, $\neq \ddagger P \leq 0.01$ and $\neq \neq \ddagger P \leq 0.001$ with respect to isoamylamine. ${ }^{\mathrm{C}} P \leq 0.001$ compares infant formulas (IF) with different days of breast milk. $P$ values calculated by non-parametric test.

compared with day $15(z=-3 \cdot 21, P=0 \cdot 03, r-0 \cdot 71)$ and day 30 $(z=-3 \cdot 23, P=0.024, r-0.77)$. Spermidine (Fig. 1(B)) increased when values of day 0 were compared with day $5(z=-3 \cdot 19$, $P=0.029, r-0 \cdot 82)$, day $7(z=-4 \cdot 14, P=0.001, r-0.84)$, day $15(z=-4 \cdot 22, P=0.001, r-0.94)$ and day $30(z=-3.77$, $P=0.003, r-0.89)$; and values of day 1 were compared with day $7(z=-3.92, P=0.002, r-0.78)$, day $15(z=-4 \cdot 01$, $P=0.001, r-0.87)$ and day $30(z=-3.56, P=0.008, r-0.82)$ and of day 3 to day $15(z=-3.07, P=0.046, r-0.69)$. Spermine (Fig. 1(C)) also increased after day 0 when compared with day $5(z=-3.28, P=0.022, r-0.88)$, day $7(z=-3.79$, $P=0.003, r-0.79)$, day $15(z=-3.43, P=0.013, r-0.81)$ and day $30(z=-3.59, P=0.007, r-0.87)$. Isoamylamine (Fig. 1(D)) significantly increased when day 0 was compared with day $7(z=-3.64, P=0.006, r-0.74)$ and day 1 with day $7(z=-4.32, P<0.001, r-0.94)$ and with day $15(z=-3.57$, $P=0.008, r-0.84)$ (Supplementary Table S1).

The values of most amines were higher in breast milk compared with infant formulas, when they were compared with each group of days. A large effect was observed from day 5 for putrescine (Fig. 1(A)) $(U=0, z=-2.56, P=0.011, r-0.81, n 10)$ and from day 0 for spermidine (Fig. 1(B)) $(U=4, z=-2 \cdot 82$, $P=0.005, r-0.71, n 16)$ and spermine (Fig. 1(C)) $(U=0$, $z=-3.18, P=0.001, r-0.82, n 15)$. The values were lower for isoamylamine (Fig. 1(D)) in breast milk compared with infant formula on day $0(U=11, z=-2.06, P=0.039, r-0.52, n 16)$ and day $1(U=5, z=-2 \cdot 81, P=0 \cdot 005, r-0 \cdot 68, n 17)$ (Supplementary Table S1).

The analysis of the biogenic amines in the different stages of the breast milk showed differences in the three stages: colostrum $(H(3)=72.16, \quad P<0.001, \quad n$ 89), transitional $(H(3)=67.78$, $P<0.001, n$ 82) and mature $(H(3)=50.21, P<0.001, n 59)$.
Putrescine (Fig. 2(A)) was found to be the least present amine in the three stages when compared with isoamylamine (Fig. 2(D)) (colostrum: $z=8.46, P<0.001, r 1.27$; transitional: $z=8.17, P<0.001, r 1.29$; mature: $P<0.001, r 1.28)$; spermidine (Fig. 2(B)) (colostrum: $z=-3.87, P=0.001, r-0.58$; transitional: $z=-4.4, P<0.001, r-0.7$; mature: $z=-4.09, P<0.001, r-0.75$ ); and spermine (Fig. 2(C)) (colostrum: $z=-4.58, P<0.001, r-0.69$; transitional: $z=-3.54, P=0.002, r-0.56)$; mature: $z=-2.82$, $P=0.029, r-0.52$ ). Spermidine (Fig. 2(B)) (colostrum: $z=4.68$, $P<0.001, r 0.706$; transitional: $z=3.87, P=0.001, r 0.597$; mature: $z=2.89, P=0.023, r 0.53$ ) and spermine (Fig. 2(C)) (colostrum: $z=3.88, \quad P=0.001, \quad r 0.578$; transitional: $z=4.75, \quad P<0.001$, $r$ 0.73; mature: $z=4.04, P<0.001, r 0.75)$ were also lower than isoamylamine (Fig. 2(D)) (Supplementary Table S2).

The values of each amine were analysed (KruskalWallis test) to determine the change during the various breast milk stages. This showed significant differences between the stages (colostrum, transitional and mature): putrescine (Fig. 2(A)) $(H(2)=15.95, P<0.001, n 56)$, spermidine (Fig. 2(B) $)(H(2)=$ $27 \cdot 25, P<0.001, n$ 59), spermine (Fig. $2(\mathrm{C}))(H(2)=10 \cdot 57$, $P=0.005, n 57)$ and isoamylamine (Fig. 2(D) $)(H(2)=20.62$, $P<0.001, n 58$ ). Mann-Whitney tests (pairwise comparisons with adjusted $P$ values) showed that putrescine (Fig. 2(A)) increased in the mature stage when compared with colostrum $(z=-3.96$, $P<0.001, r-0.65)$ and transitional stages $(z=-2.73, P=0.019$, $r-0.47$ ), spermidine (Fig. 2(B)) increased in the transitional $(z=-4.08, P<0.001, r-0.62)$ and mature stage $(z=-4.7$, $P<0.001, r-0.76)$ when compared with colostrum; and the concentrations of spermine (Fig. 2(C)) also increased in the transitional $(z=-2.44, P=0.044, r-0.37)$ and mature stages $(z=-3$, $P=0.008, r-0.5)$ when compared with colostrum. Isoamylamine (Fig. 2(D)) concentrations were also higher in the transitional 

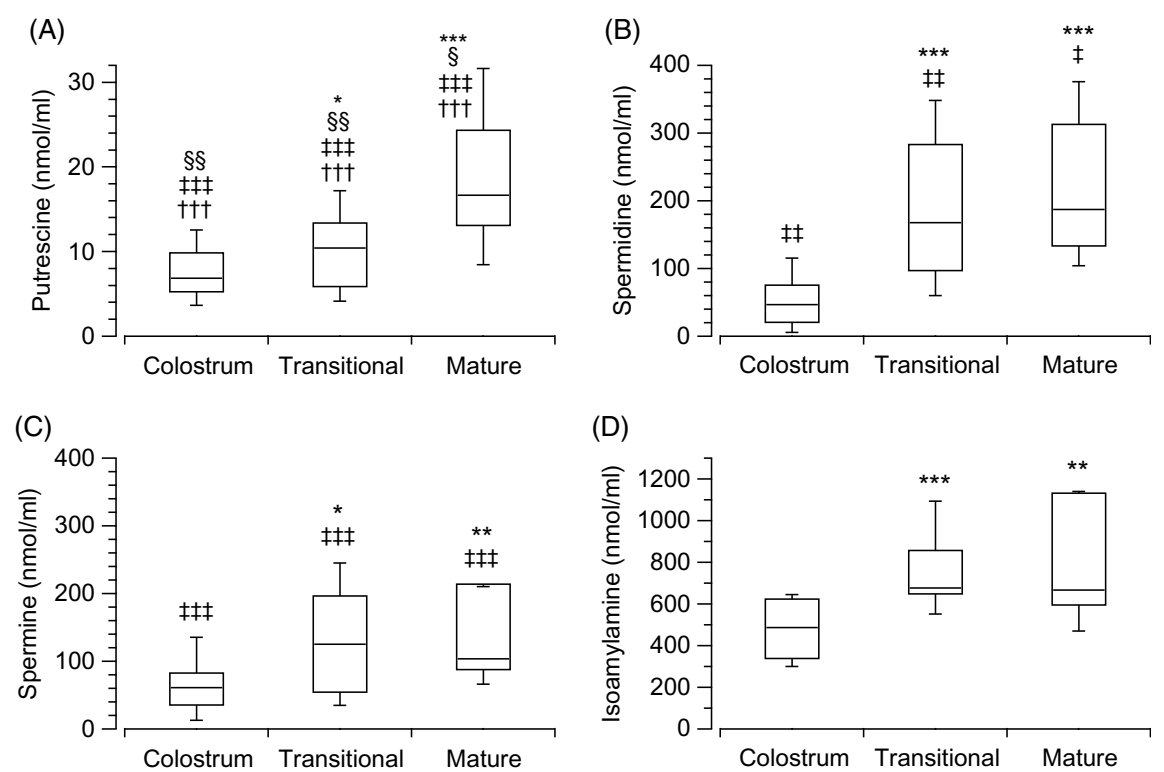

Fig. 2. Box and whisker plots of the values (nmol/ml) of putrescine (A), spermidine (B), spermine (C) and isoamylamine (D) in the stages of breast milk (colostrum, transitional and mature). ${ }^{*} P \leq 0.05,{ }^{* *} P \leq 0.01$ and ${ }^{* * *} P \leq 0.001$ in comparison with colostrum; $\ddagger \ddagger P \leq 0.01$ and $\ddagger \ddagger \ddagger P \leq 0.001$ with respect to isoamylamine; $\dagger \dagger \dagger P \leq 0.001$ with respect to spermidine and $\S P \leq 0.05$ and $\S \S P \leq 0.01$ with respect to spermine on the same stage. $P$ values calculated by non-parametric test.

$(z=-4 \cdot 33, P<0 \cdot 001, r-0 \cdot 66)$ and the mature stage $(z=-3 \cdot 15$, $P=0.005, \quad r-0.52)$ compared with the colostrum stage (Supplementary Table S2).

\section{Correlation between the amines present in human breast milk or infant formulas}

In breast milk, the values of putrescine correlated positively and significantly with spermidine $\left(r_{s}=0.53, P<0.001, n\right.$ 65) and spermine $\left(r_{s}=0.54, P<0.001, n 62\right)$, and the values of spermidine correlated positively and significantly with spermine $\left(r_{s}=0.93\right.$, $P<0.001, n$ 65). The concentration of isoamylamine correlated positively and significantly with spermidine $\left(r_{s}=0.55, P<0.001\right.$, $n$ 67) and spermine $\left(r_{s}=0.4, P<0.001, n 64\right)$. In infant formulas, no significant correlations exist between the different amines.

\section{Content of biogenic amines in the faeces of newborns}

In the meconium (day 0), no differences were observed in the content of each polyamines depending on the type of feeding (Fig. 3). Although differences were observed when the content of polyamines were compared in each type of feeding (breast-fed: $H(2)=37 \cdot 98, P<0 \cdot 001, n 60$; infant formula feed: $H(2)=27.85, \quad P<0.001, n 42)$. In both cases, putrescine (Fig. 3(A)) was the polyamine present at the highest concentration (Mann-Whitney tests with adjusted $P$ values), showing large effect size when compared with spermidine (Fig. 3(B)) $(z=5 \cdot 38$, $P<0.001, r 0.85$ and $z=4.88, P<0.001, r 0.92$, respectively, for each type of feeding) and spermine (Fig. 3(C)) $(z=5 \cdot 3$, $P<0 \cdot 001, r 0 \cdot 83$ and $z=4 \cdot 18, P=0, r 0 \cdot 79$, respectively, for each type of feeding). Considering all the amines, the content of isoamylamine (Fig. 3(D)) was higher than putrescine (Fig. 3(A)) in breast milk feeding $(U=66, z=-3.63, P<0.001, r-0.57$, $n$ 40) and in infant formula feeding ( $U=40, z=-2 \cdot 67$, $P=0.008, r-0 \cdot 5, n 28)$. No differences were observed in the content of isoamylamine (Fig. 3(D)), cadaverine (Fig. 3(E)) and tyramine (Fig. 3(F)) between both types of feeding.

The concentrations of polyamines in faeces increased over time, with significant differences across the groups of days in both types of feeding (expressed for breast-fed and infant formula-fed, respectively, putrescine (Fig. 3(A)): $H(5)=28.94$, $P<0.001(n 79)$ and $H(5)=22.84, P<0.001$ ( $n$ 55), spermidine (Fig. 3(B)): $H(5)=24.96, P<0.001(n$ 79) and $H(5)=25.79$, $P<0.001$ ( $n$ 55), and spermine (Fig. $3(\mathrm{C})$ ): $H(5)=18.07$, $P=0.003(n$ 79) and $H(5)=18.52, P=0.002(n$ 55)). Pairwise comparisons (Mann-Whitney tests with adjusted $P$ values) of polyamines in the faeces of breast milk-fed newborns showed that putrescine (Fig. 3(A)) content was highest on day 3 $(z=-3.91, \quad P=0.001, \quad r-0.66)$ and day $7(z=-3.61$, $P=0.004, r-0.68)$ from the start of breast-feeding as well as for these days when compared with the 1st day $(z=-3.54$, $P=0.006, r-0.6$ and $z=-3 \cdot 3, P=0.014, r-0.64$, respectively). The values of spermidine (Fig. 3(B)) were highest on day 3 $(z=-4.04, \quad P<0.001, \quad r-0.68)$ and day $30 \quad(z=-3 \cdot 6$, $P=0.004, r-0 \cdot 71)$ when compared with day 0 . No significant differences were observed for spermine (Fig. 3(C)) across the groups of days. Similarly, in newborns fed with infant formula the concentration of putrescine (Fig. 3(A)) was highest on day $3(z=-3.57, P=0.005, r-0.65)$ and day $7(z=-3.62$, $P=0.004, r-0.83)$ after birth with respect to day 0 , which decreased subsequently. Spermidine (Fig. 3(B)) increased on day $3(z=-4, P<0.001, r-0.73)$ and also significantly increased on days $7(z=-4.41, P<0.001, r-1 \cdot 01), 15$ $(z=-3 \cdot 16, P=0.023, r-0.75)$ and $30(z=-3.93, P=0.001$, $r$-0.9) when compared with day 0 and increased on day 7 when compared with day $1(z=-3 \cdot 12, P=0 \cdot 027, r-0 \cdot 78)$. Spermine (Fig. 3(C)) also increased on day $3(z=-3.49$, $P=0.007, r-0.64$ ) when compared with day 0 (Supplementary Tables S3 and S4). 
(A)

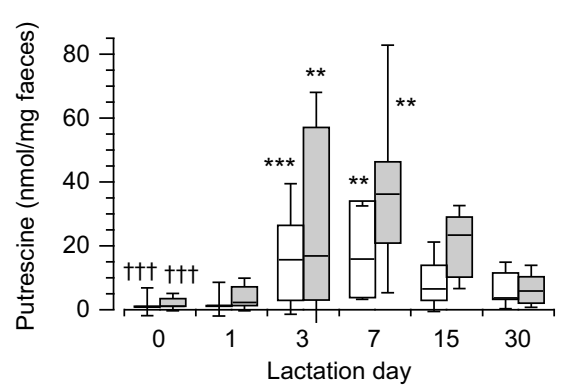

(D)

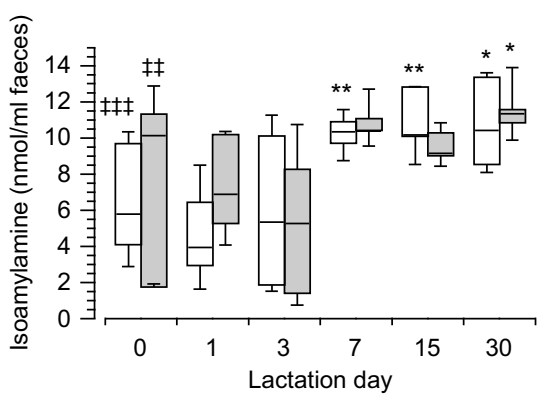

(B)

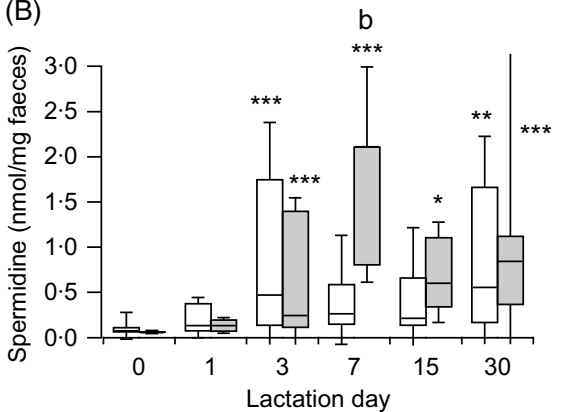

(E)

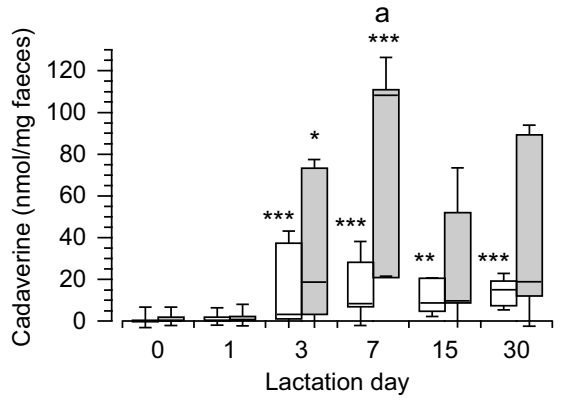

(C)

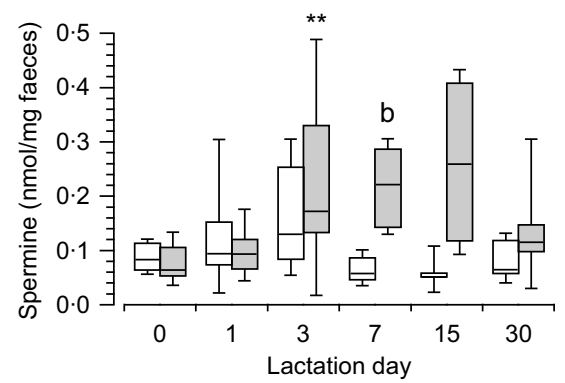

(F)

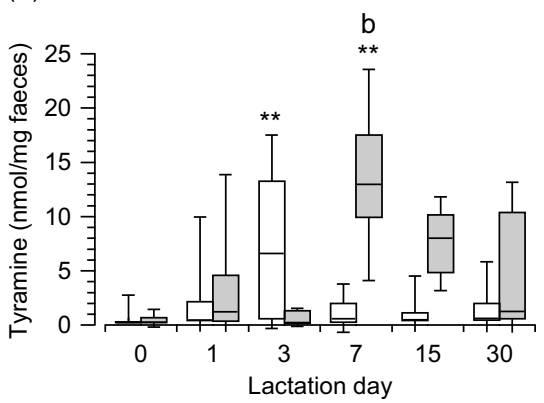

Fig. 3. Box and whisker plots of the values in $\mathrm{nmol} / \mathrm{mg}$ of faeces for putrescine (A), spermidine (B), spermine (C), isoamylamine (D), cadaverine (E), and tyramine ( $F)$ in breast (white) or infant formula (grey) fed from the day of birth (day 0 ) to day 30 postpartum. ${ }^{\star} P \leq 0.05$, ${ }^{* \star} P \leq 0.01$ and ${ }^{* \star \star} P \leq 0.001$ in comparison with day 0 ; $† \dagger \dagger P \leq 0.001$ with respect to spermidine and spermine and $\ddagger \ddagger P \leq 0.01$ and $\ddagger \ddagger \ddagger P \leq 0.001$ isoamylamine with respect to the rest of the amines. ${ }^{\mathrm{a}} P \leq 0.05$ and ${ }^{\mathrm{b}} P \leq 0.01$ compare the content of amines in the faeces of infant formula-fed newborns with breast milk-fed newborns. $P$ values calculated by non-parametric test. $\square$, Breast milk; $\square$, infant formula.

As for polyamines, the content of the primary amines in the faeces of newborns varied over time after birth in both types of feeding (isoamylamine (Fig. 3(D)): $H(5)=24 \cdot 02, P<0 \cdot 001$ ( $n$ 79, and $H(5)=11.58, P=0.041$ ( $n$ 55); cadaverine (Fig. 3(E)): $H(5)=33.92, P<0.001(n 79)$, and $H(5)=26.25$, $P<0.001$ ( $n$ 54); tyramine (Fig. 3(F)): $H(5)=14 \cdot 8, P=0.011$ ( $n$ 79), and $H(5)=15.99, P=0.007$ ( $n$ 51), respectively, for breast milk and infant formula feeding). Pairwise comparison between groups of days in breast milk-fed newborns (MannWhitney tests with adjusted $P$ values) showed that isoamylamine (Fig. 3(D)) increased on day $7(z=-3 \cdot 22, P=0 \cdot 019$, $r-0.62)$ after birth and maintained nearly the same levels until day $15(z=-3 \cdot 44, P=0 \cdot 008, r-0 \cdot 68)$ and day $30(z=-3 \cdot 28$, $P=0.015, r-0.66)$. Cadaverine (Fig. 3(E)) increased on day $3(z=-3.94, P=0.001, r-0.74)$ and remained at around the same level on day $7(z=-4.02, P<0.001, r-0.76)$, day 15 $(z-3.74, \quad P=0.002, \quad r=-0.63)$ and day $30(z=-3.98$, $P=0.001, r-0.78)$ after birth. Tyramine (Fig. 3(F)) showed a peak increase on day $3(z=-3.78, P=0.002, r-0.64)$ with respect to day 0 . For infant formula-fed newborns (MannWhitney tests with adjusted $P$ values), isoamylamine (Fig. 3(D)) was significantly highest on day $30(z=-3 \cdot 08$, $P=0.02, r-0.67)$ with respect to day 3 after birth; cadaverine (Fig. 3(E)) increased after day $3(z=-3 \cdot 34, P=0 \cdot 012, r-0 \cdot 61)$, peaked on days $7(z=-3.89, P=0.001, r-0.89)$ and 30 $(z=-2.97, P=0.044, r-0.68)$ when compared with day 0 and also peaked on day $7(z=-3 \cdot 29, P=0 \cdot 014, r-0 \cdot 82)$ when compared with the 1st day after birth. Tyramine (Fig. 3(F)) showed a peak increase on day $7(z=-3.46, P=0.008$, $r-0.79)$ when compared with the day of birth (Supplementary Tables S3 and S4).
The magnitude of increase in the biogenic amines in faeces, over time, were significantly higher for infant formula-fed newborns compared with breast milk-fed newborns on day 7 , namely, spermidine (Fig. 3(B)) $(U=5, z=-2 \cdot 45, P=0.014$, $r-0.63, n 15)$, spermine (Fig. 3(C)) $(U=2, z=-2 \cdot 82, P=0.005$, $r-0 \cdot 73, n 15)$, cadaverine (Fig. 3(E)) $(U=8, z=-2 \cdot 08, P=0.037$, $r-0.54, n$ 15) and tyramine (Fig. 3(F)) $(U=4, z=-2.57$, $P=0.01, r-0.66, n$ 15) and also for tyramine (Fig. $3(\mathrm{~F}))$ at day $15(U=3, z=-2 \cdot 32, P=0 \cdot 021, r-0 \cdot 64, n 13)$. No statistical significance was shown for putrescine (Fig. 3(A)), whose differences were NS, and for isoamylamine (Fig. 3(D)), which was very similar in both types of feeding (Supplementary Tables S3 and S4).

The biogenic amine content in faeces was also analysed in the intervals of days considered to establish the breast milk stages (colostrum from days 1 to 5, transitional from days 6 to 14 and mature from days 15 to 30 after birth). The comparison dependent on the type of feeding showed no differences during the days of colostrum. However, during transitional stage days, spermidine $(U=11, z=-2.39, P=0.016, r-0.52, n 21)$, spermine $(U=11, z=-2 \cdot 39, P=0.016, r-0.52, n 21)$, cadaverine $(U=12, z=-2 \cdot 31, P=0 \cdot 02, r-0 \cdot 5, n 21)$ and tyramine $(U=9$, $z=-2 \cdot 56, P=0 \cdot 01, r-0 \cdot 56, n 21)$ were higher in faeces of infant formula-fed newborns compared with those fed breast milk. Spermine ( $U=26, z=-2 \cdot 83, P=0.004, r-0.54, n 27)$ and tyramine ( $U=41, z=-2.06, P=0.039, r-0.39, n$ 27) were still higher in the faeces of those infant formula fed during the days of mature stage (Supplementary Table S5).

The content of the biogenic amines in faeces varied significantly across the days of the stages, depending on the type of feeding: spermine in breast-fed $(H(2)=10 \cdot 09, P=0 \cdot 006, n$ 68) 
(A)

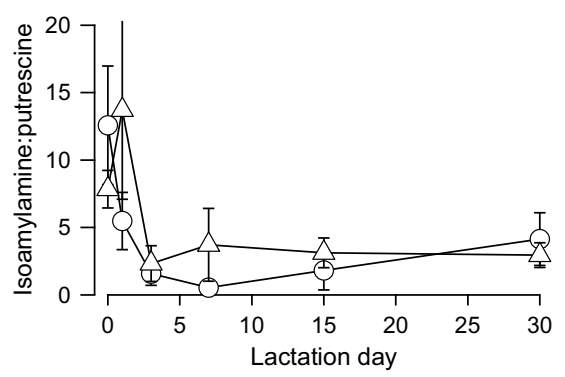

(D)

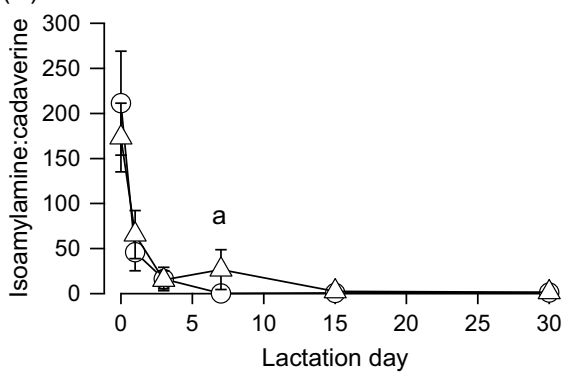

(B)

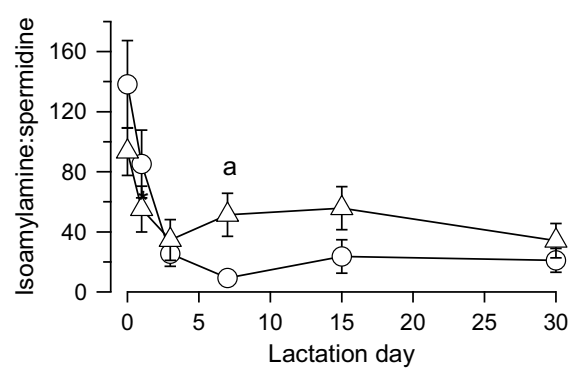

(C)

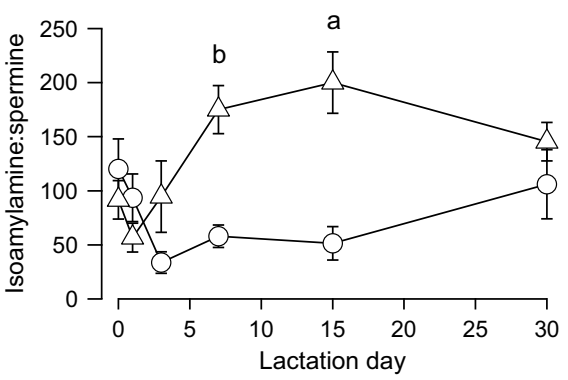

Fig. 4. Ratios of isoamylamine concentration to putrescine (A), spermidine (B), spermine (C), cadaverine (D), or tyramine (E) in the faeces of breast milk-fed (-o-) and infant formula-fed $(--)$ newborns, from the day of birth (day 0$)$ to day 30 postpartum. Values are means, with standard errors represented by vertical bars. ${ }^{a} P \leq 0.05$ and ${ }^{\mathrm{b}} P \leq 0.01$ compare infant formulas with different days of breast milk. $P$ values calculated by non-parametric test.

and spermidine in the infant formula fed $(H(2)=12 \cdot 38$, $P=0.002, \quad n$ 41), and isoamylamine in both types $(H(2)=16.96, P<0.001, n 68$ and $H(2)=12, P=0.002, n 41$, respectively) and cadaverine $(H(2)=8 \cdot 89, P=0.012, n 68$ and $H(2)=8.63, P=0.013, n 41$, respectively). In the faeces of breast-fed newborns, the content of spermine decreased significantly in the mature stage days when compared with colostrum days $(z=2.94, P=0.01, r 0.407)$, while isoamylamine significantly increased in the transitional $(z=-2.65, P=0.024$, $r-0.37)$ and mature stage days $(z=-3.86, P<0.001$, $r-0.53)$ with respect to colostrum as well as cadaverine in the mature stage days $(z=-2 \cdot 68, P=0 \cdot 022, r-0 \cdot 37)$. For infant formula-fed newborns, spermidine increased in transitional $(z=-2.95, P=0.01, r-0.52)$ and mature $(z=-2.47, P=0.041$, $r-0 \cdot 41)$ stages with respect to colostrum days and also increased isoamylamine in the transitional $(z=-2 \cdot 38, P=0.05, r-0.4)$ and mature stage days $(z=-2.94, P=0.01, r-0.52)$ and cadaverine in the transitional stage days $(z=-2 \cdot 74, P=0 \cdot 019, r-0 \cdot 48)$. No significant modifications were observed for the remaining biogenic amines (Supplementary Table S5).

Ratios of isoamylamine with the other amines in faeces of both types of feeding

The finding that isoamylamine content and variations over time were similar in the faeces of both types of feeding led us to use this amine as a reference to normalise the other amines as a ratio. All the ratios decreased, with an analogous time constant $(1.64 \pm 0.16 \mathrm{~d})$, from the day after birth until day 5 , without significant differences between breast milk- and infant formulafed newborns (Fig. 4). Then the ratios of isoamylamine:spermidine (Fig. 4(B)) $(U=5, z=-2.45, P=0.014, r-0.63, n$ 15),
(E)

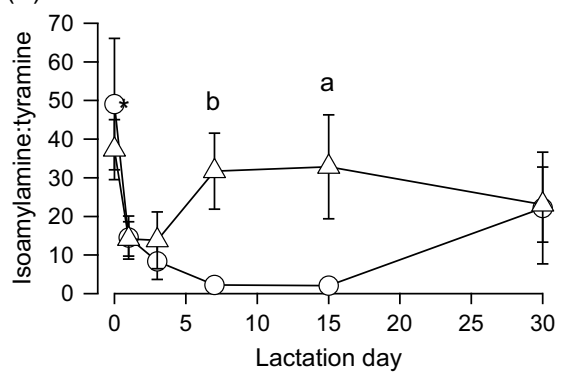

isoamylamine:spermine (Fig. $4(\mathrm{C}))(U=2, z=-2 \cdot 82, P=0 \cdot 005$, $r-0.73, n$ 15), isoamylamine:cadaverine (Fig. 4(D)) $(U=8$, $z=-2 \cdot 08, P=0.037, r-0.54, n 15)$ and isoamylamine:tyramine (Fig. 4(E)) ( $U=4, z=-2 \cdot 57, P=0.01, r-0 \cdot 66, n 15)$ increased significantly on day 7 but not of isoamylamine:putrescine (Fig. 4(A)). The ratios of isoamylamine:spermine (Fig. 4(C)) ( $U=2, z=-2 \cdot 47, P 0 \cdot 014, r-0 \cdot 68, n$ 13) and isoamylamine: tyramine (Fig. 4(E)) $(U=3, z=-2 \cdot 32, P=0 \cdot 021, r-0 \cdot 64, n 13$ ) remained in the same level on day 15 .

\section{Correlation between biogenic amines in faeces of the newborns fed human breast milk and infant formulas}

In the faeces of breast-fed newborns, the content of putrescine correlated positively and significantly with spermidine ( $r_{s} 0.62, P=0.001, n$ 81) and spermidine with spermine ( $\left.r_{s}=0.35, P=0.001, n 81\right)$. Putrescine correlated significantly with cadaverine $\left(r_{s}=0.78, P=0.001, n 81\right)$ and tyramine $\left(r_{s}=0.52, P=0.001, n 76\right)$. In addition, spermine correlated negatively and significantly with isoamylamine $\left(r_{s}=-0.52\right.$, $P=0.001, n 81)$ and positively with tyramine $\left(r_{s}=0.51\right.$, $P=0 \cdot 001, n 76)$. Cadaverine positively correlated with tyramine $\left(r_{s}=0.51, P=0.001, n 76\right)$ and isoamylamine $\left(r_{s}=0 \cdot 24\right.$, $P=0.03, n 81)$.

In infant formula-fed newborns, putrescine correlated significantly with spermidine $\left(r_{s}=0.7, P<0.001, n 55\right)$ and spermine $\left(r_{s}=0.52, P<0.001, n 55\right)$, and spermidine with spermine $\left(r_{s}=0.7, \quad P<0.001, n\right.$ 55). Putrescine, spermidine and spermine correlated positively with cadaverine (respectively, $r_{s}=0.81, P<0.001, n 54 ; r_{s}=0.73, P<0.001, n 54$; and cadaverine $\left.r_{s}=0.52, P<0.001, n 54\right)$ and tyramine (respectively, $r_{s}=0.64, P<0.001, n 51 ; r_{s}=0.64, P<0.001, n 51 ;$ and 
$\left.r_{s}=0.58, P<0.001, n 51\right)$. Cadaverine correlated positively with tyramine $\left(r_{s}=0.49, P<0.001, n 50\right)$.

Correlation between polyamines and primary amines in faeces of the newborns with respect to the type of feeding, breast milk or infant formula feeding

In breast-fed newborns, the concentration of putrescine in breast milk correlated positively with the content of isoamylamine in faeces $\left(r_{s}=0 \cdot 42, P=0 \cdot 007, n 40\right)$. The concentration of spermidine in breast milk correlated positively with the content of putrescine $\left(r_{s}=0.46, P=0.002, n 42\right)$, spermidine $\left(r_{s}=0.43\right.$, $P=0.005, n 42)$, isoamylamine $\left(r_{s}=0.58, P<0.001, n 42\right)$ and cadaverine $\left(r_{s}=0 \cdot 47, P=0 \cdot 002, n 42\right)$ in faeces. Similarly, spermine in breast milk correlated with cadaverine in faeces $\left(r_{s}=0.43\right.$, $P=0.005, n 40)$. Also isoamylamine in breast milk correlated positively with the content in faeces of this amine $\left(r_{s}=0.59\right.$, $P<0.001, n 41)$ and of putrescine $\left(r_{s}=0.4, P=0.01, n 41\right)$, cadaverine $\left(r_{s}=0.41, P=0.008, n 41\right)$, spermidine $\left(r_{s}=0.36\right.$, $P=0.023, n 41)$ and spermine $\left(r_{s}=-0.47, P=0.002, n 41\right)$.

In faeces of infant formula-fed newborns, the content of isoamylamine in faeces correlated positively with the concentration of putrescine $\left(r_{s}=0.4, P=0.002, n 55\right)$ and spermidine $\left(r_{s}=0.53, P<0.001, n 55\right)$ in the breast milk.

When the analysis was restricted to the days included in the transitional and mature stages, the content of spermine in faeces of breast-fed newborns correlated positively with the concentration of spermidine $\left(r_{s}=0.47, P=0.037, n 20\right)$ and spermine $\left(r_{s}=0.45, P=0.048, n 20\right)$ in breast milk. Also in the infant formula-fed newborns, the isoamylamine $\left(r_{s}=0.6, P=0.025\right.$, $n$ 14) and tyramine $\left(r_{s}=-0 \cdot 61, P=0.022, n\right.$ 14) contents in faeces were correlated positively and negatively, respectively, with the concentration of spermidine in the infant formulas.

\section{Prediction of breast milk or infant formula feeding based on faeces amine content}

Regression logistic analysis was used to determine the possibility of using amines to predict the types of newborn feeding, for the days included in transitional and mature stages where differences in amines were found. The model contains two independent variables cadaverine and tyramine in $\mathrm{nmol} / \mathrm{mg}$ of faeces, statistically significant $\chi^{2}(2, n 48)=27 \cdot 47, P<0 \cdot 001$, indicating that the model was able to distinguish between breast milk and infant formula. The model correctly classified $85.4 \%$ of the cases (Cox and Snell $R^{2}: 34 \cdot 7 \%$ and Nagelkerke $R^{2}: 36 \cdot 3 \%$ ). Only two of the independent variables made a unique statistically significant contribution to the model (cadaverine and tyramine in faeces). The strongest predictor of the feeding type was the content of tyramine in faeces, recording an OR of 1.29 , then cadaverine in faeces, 1.027. The formula to predict the type of feeding was $Y_{\text {breast milk/infant formula }}=-2 \cdot 837+$ (cadaverine $(\mathrm{nmol} / \mathrm{mg}$ faeces $) \times 0.026)+$ (tyramine $(\mathrm{nmol} / \mathrm{mg}$ faeces $)$ $0 \cdot 258)$, with a cutoff value of 0.5 .

\section{Discussion}

The results of the study showed that the contents of polyamines and primary amines in the faeces of newborns, during the first $30 \mathrm{~d}$ after birth, are determined by the feeding type, that is, breast milk or infant formula, but it is not related to the amount ingested. The differences in the content may be related to the distinct presence of amine-producing bacteria, which polyamines can help establish.

The first and only source of nutrition in newborns is breast milk or, alternatively, feeding with infant formulas. Both feeding types provide several components, polyamines being one of them, which crucial to the development of the gastrointestinal tract and the establishment of microbiota and immune system, with difficult results. Consequently, it is a matter of interest to determine the influence of variations in the amounts of polyamines provided on their intestinal content (subsidiary studied in faeces).

The measurement of polyamines in human breast milk showed that at birth and for the first $3 \mathrm{~d}$ the most concentrated were spermidine and spermine. All then increased up to the 2nd week postpartum, with a tendency to decrease afterwards. Spermidine was the most concentrated polyamine, reaching roughly four to five times the initial concentration. When referring to the stages of breast milk, the concentrations of polyamines were lower in the colostrum stage, putrescine being the least concentration, and then increased in the transitional and mature stages. Overall, these data are in line with previous reports, showing increases in polyamines over time, though quantitative differences are present ${ }^{(34,50,51)}$. These have been explained by the lifestyle, type of diet, the characteristics of the women and perinatal factors, as mentioned above, and by the different methods used to extract the biogenic amines of the samples among diverse studies. To minimise the influential factors as much as possible, our study was performed in the same geographical area on Caucasian, non-obese, healthy women, who gave birth after the 38th week of pregnancy to vaginally delivered infants (to avoid differences in the polyamines and of the acquired microbiota ${ }^{(37,52-54)}$ ), and using both types of feeding methods. The study demonstrated, as has previously been observed, the existence of individual variations in polyamine content (including spermine, in some studies less susceptible to environmental influences ${ }^{(37,55)}$ ) found in the breast milk of different mothers, at least partly related to different human milk microbiota profiles ${ }^{(37,56,57)}$. It should be noted that despite the similar characteristics and socio-cultural environment of the mothers and expecting similar profile in breast milk microbiota within the geographical area ${ }^{(53,55,56)}$, they could have had dietary habits (not investigated in the study) that may have affected the content of polyamines in breast milk, which may have had physiological consequences.

The comparison of the polyamine content in both types of feeding showed that spermidine and spermine were significantly lower in infant formulas compared with breast milk for the entire 1 st month. For putrescine, the changes were observed after the 1st week postpartum, similar to previous reports ${ }^{(50,51,58,59)}$. In breast milk, but not in infant formulas, there existed linear positive correlations between putrescine and spermidine, between putrescine and spermine and between spermidine and spermine. These correlations may reflect common sources of production. Infant formulas are given at a fixed composition of constituents, which does not match the variations over time observed in breast milk ${ }^{(51)}$. 
The primary amine isoamylamine was identified in breast milk and infant formulas, and no other biogenic amines were present. In infant formula biogenic amines were infrequently detected or were present at very low levels ${ }^{(60)}$. Its presence is associated with poor hygienic quality in non-fermented foods ${ }^{(61)}$. Therefore, this indicates that our samples were correctly preserved. It is interesting that isoamylamine in breast milk also varies with time, increasing until the 1st week after delivery. The first days were less concentrated than in infant formulas and, subsequently, were similar. Isoamylamine presence in breast milk and its sources have not been previously mentioned, its sources are also unknown in other human tissues where it has been found ${ }^{(62)}$. It has been reported in mice kidney that expressed a specific decarboxylase of leucine forming isoamylamine $^{(63)}$ and is also produced by bacteria ${ }^{(64-67)}$. Among them, Lactobacillus plantarum (considered a probiotic species by the European Food Safety Authority) and Enterococcus faecalis are present in breast milk ${ }^{(68-70)}$. These bacteria produce isoamylamine, at least in cheese $\mathrm{e}^{(67)}$. In breast milk, but not in infant formulas, isoamylamine correlated positively with the concentrations of spermidine and spermine, suggesting the existence of producer or degrader factors in common with the polyamines, affecting or being affected by them. This matter has not yet been studied in human milk.

The determination of polyamines and primary amines in the meconium of newborns revealed that at birth no differences were observed in the content of amines between the two feeding groups. This appears to demonstrate their homogeneity, which was taken into consideration when choosing term pregnancies by giving natural birth, which may affect newborns' microbiota $^{(53,71)}$. The average age and number of deliveries of the mothers and the sex of the newborns were also similar. In the meconium, the most important amine was isoamylamine, and putrescine of the polyamines. Histamine was not present, or could have also been below the detection levels, in the faeces of newborns.

After birth, nutrition is one of the sources of polyamines, contributing to those produced by the organs of the digestive system and the microbiota. For primary amines (except for isoamylamine, present in breast milk), the main source is produced by intestinal bacteria, which may vary depending on the feeding type of newborns ${ }^{56,57,72)}$. Through breast milk, the mother's microbiota is transferred and helps in establishing the newborns' own microbiota. The commensal bacteria and the antimicrobial factors produced by the microbiota or by compounds present in human milk (bioactive or acting as probiotics) are important. In addition, the intestinal absorption of polyamines determines its intraluminal content.

Our results showed that in the faeces of newborns, polyamines and the primary amines increased differently from day 3 after birth, the peak amplitude being in infant formula fed on day 7 , which is greater than that in breast-fed (except for putrescine, no significant change, and isoamylamine, that followed similar evolution over time in both groups). This did not match the quantitative changes observed over time in breast milk and infant formula. In fact, it was the opposite, suggesting that other factors, apart from nutrition, are involved. However, an association between some polyamines and biogenic amine content seemed to exist in faeces, with differences based on the type of feeding. Thus, during the days of transitional and mature stages of breast milk, positive correlations existed between the content of isoamylamine and tyramine in the faeces with the presumable intake of spermidine by breast milk. While in the faeces of infant formula-fed children, the content of cadaverine correlated significantly to spermine intake, and the content of spermine in faeces with the content of spermidine and spermine in the infant formulas. The analysis of the ratios of isoamylamine to the remaining amines showed that they decreased with a time constant of about $2 \mathrm{~d}$, and after day 5 these were mostly stable, independent of the type of nutrition, suggesting an equilibrium in the homeostatic factors determining their concentrations. The only exceptions were in the faeces of infant formula-fed newborns during the 2nd week; there were an increase in the ratios of isoamylamine:spermidine and, especially, isoamylamine:spermine and isoamylamine:tyramine, in comparison with breast-fed infants. These differences indicate that the homeostases of biogenic amines in the faeces are complex and determined by more factors than the amount ingested. But were reduced by the end of the 1st month.

In adult faecal microbiota, diverse micro-organisms have been associated with the production of biogenic amines depending on the strain and the culture conditions, when grown in the presence of a cocktail of amino acids (not leucine) or amines (polyamines, cadaverine, tyramine and histamine, but not isoamylamine). In the presence of these amines, overall a decrease in the production of putrescine and cadaverine by most Grampositive bacteria and of tyramine mostly by Enterococcus strains were observed. While increases in the production of spermidine, spermine and histamine in several Gram-positive and Gramnegative strains have been demonstrated ${ }^{(73)}$. It is not easy to extrapolate this in vitro experiment, a cocktail with a fixed concentration of amines $(0.03 \%)$, with the variable conditions of the nutritional factors ${ }^{(72,74)}$ and microbiota ${ }^{(56,57,75,76)}$ found in newborns during lactation. But it suggests that the ingested polyamines and isoamylamine may contribute to facilitating or limiting the growth of different faecal bacteria strain producers or degraders of biogenic amines. An inter-individual variability exists in the microbiota within breast-fed newborns and between the type of feeding ${ }^{(57,77,78)}$. Among the bacteria associated with infant formula feeding, Streptococcus and Enterococcus species were discriminatory ${ }^{(57)}$. Some strains of Enterococcus faecalis are producers of putrescine, tyramine and cadaverine ${ }^{(73,79,80)}$, Escherichia coli also produces cadaverine ${ }^{(73)}$. The presence of these bacteria in faeces might explain the higher content of putrescine and cadaverine found in infant formula-fed infants.

In addition, human breast milk feeding facilitates the histological and functional maturation of the epithelium and intestinal villi and of the immune system after birth and may also decrease inflammation caused by the introduction of oral feeding in infants ${ }^{(81)}$. Among other aspects, polyamines are involved in these effects ${ }^{(26,82)}$. Intestinal maturation is going to presumably increase the capability of absorption, decreasing the intestinal content of polyamines and of amino acids, of which leucine, lysine and tyrosine are precursors of isoamylamine, cadaverine and tyramine, respectively, altering their concentration in faeces. The greater concentration of polyamines and primary amines in 
faeces, among other mechanisms, could explain the reduction in the frequency of stools and the alteration in faecal consistency in infant formula feeding. This seems to suggest the decrease in motility shown on segmentation contractions and peristalsis of isolated intestinal tissues ${ }^{(30,31)}$ (with effects on the intestinal digestion and absorption of nutrients) and the gastrointestinal benefits for children's diarrhoea, when the solutions for oral rehydration or infant formulas were supplemented with polyamines $^{(83)}$. These effects could be receptor dependent, being involved in the crosstalk between the microbiota and intestinal function $^{(84)}$, since trace amine-associated receptors for cadaverine, isoamylamine and tyramine have been reported in the gut ${ }^{(32)}$.

During the 2nd week, results showed a quantitative difference in the content of polyamines, cadaverine and tyramine in faeces, depending on the type of feeding. Additional studies should clarify the factors that determine these differences: nutritional, microbiota and/or effects on intestinal maturation and absorption. The concentrations of polyamines during lactation or when giving infant formulas could be adjusted and then evaluated for their influence on the biogenic amines in faeces. The determination of cadaverine and tyramine in faeces may be useful as biological markers to determine the polyamines supplied during the first 2 weeks after birth, since they may predict, with $85 \%$ of accuracy, the type of feeding method given.

It also seems important to determine in faeces the relevant amine or amines, and its concentrations, with the corresponding histological and metabolic gastrointestinal effects as well as identify the micro-organisms that produce the biogenic amines found in the faeces that may provide information about potential differences in the microbiota. In newborns, the experimental studies supplementing polyamines orally should be considered carefully due to safety issues ${ }^{(85,86)}$. Although it will be of interest to determine whether supplementing polyamines may result in faeces presenting similar patterns to breast milk feeding. In the meantime, concentrations devoid of undesirable effects should be established, since cytotoxicity of biogenic amines has been reported $^{(87,88)}$

The present results may have some limitations in inferring to populations since the sample was neither selected randomly nor the evolution over time applies to the same subjects. These were independent samples, which may have introduced some biases. In any case, it should be noted that participants were recruited consecutively (following the inclusion criteria) through the public health system, which serves most of the population in the corresponding geographical area, regardless of their socio-economic and cultural differences.

\section{Acknowledgements}

The authors highly appreciate the generosity of the families providing us with the samples used in the study.

This work was supported by Grants from the Universidad de Oviedo (SV-UNOV-10-MA-2, UNO-11-MA-13), Spain.

All authors participated in the design of the study and different aspects to carry it out, mainly L. S., M. M. L. and M. S. analysed the data, and M. S. wrote the article.

There are no conflicts of interest.

\section{Supplementary material}

For supplementary material/s referred to in this article, please visit https://doi.org/10.1017/S0007114519001879

\section{References}

1. Holscher HD, Bode L \& Tappenden KA (2017) Human milk oligosaccharides influence intestinal epithelial cell maturation in vitro. J Pediatr Gastroenterol Nutr 64, 296-301.

2. Turfkruyer M \& Verhasselt V (2015) Breast milk and its impact on maturation of the neonatal immune system. Curr Opin Infect Dis 28, 199-206.

3. Boix-Amorós A, Puente-Sánchez F, du Toit E, et al. (2019) Mycobiome profiles in breast milk from healthy women depend on mode of delivery, geographic location, and interaction with bacteria. Appl Environ Microbiol 85, e02994-e02918.

4. Parigi SM, Eldh M, Larssen P, et al. (2015) Breast milk and solid food shaping intestinal immunity. Front Immunol 6, 415.

5. WHO (2000) Effect of breastfeeding on infant and child mortality due to infectious diseases in less developed countries: a pooled analysis. WHO collaborative study team on the role of breastfeeding on the prevention of infant mortality. Lancet 355, 451-455.

6. Armstrong J \& Reilly JJ (2002) Breastfeeding and lowering the risk of childhood obesity. Lancet 359, 2003-2004.

7. Forbes JD, Azad MB, Vehling L, et al. (2018) Association of exposure to formula in the hospital and subsequent infant feeding practices with gut microbiota and risk of overweight in the first year of life. JAMA Pediatr 172, e181161.

8. Roberts SB (2001) Prevention of hypertension in adulthood by breastfeeding? Lancet 357, 406-407.

9. Victora CG, Horta BL, Loret de Mola C, et al. (2015) Association between breastfeeding and intelligence, educational attainment, and income at 30 years of age: a prospective birth cohort study from Brazil. Lancet Glob Health 3, e199-e205.

10. Kibe R, Kurihara S, Sakai Y, et al. (2014) Upregulation of colonic luminal polyamines produced by intestinal microbiota delays senescence in mice. Sci Rep $\mathbf{4}, 4548$.

11. Evenhouse E \& Reilly S (2005) Improved estimates of the benefits of breastfeeding using sibling comparisons to reduce selection bias. Health Serv Res 40, 1781-1802.

12. Girard LC, Doyle O \& Tremblay RE (2017) Breastfeeding, cognitive and noncognitive development in early childhood: a population study. Pediatrics 139, e20161848.

13. Colen CG \& Ramey DM (2014) Is breast truly best? Estimating the effects of breastfeeding on long-term child health and wellbeing in the United States using sibling comparisons. Soc Sci Med 109, 55-65.

14. Der G, Batty GD \& Deary IJ (2006) Effect of breast feeding on intelligence in children: prospective study, sibling pairs analysis, and meta-analysis. BMJ 333, 945.

15. Raissian KM \& Su JH (2018) The best of intentions: prenatal breastfeeding intentions and infant health. SSM Popul Health 5, 86-100.

16. Pilkington H (2003) Should we encourage exclusive breastfeeding at all cost? Lancet 362, 247.

17. American Academy of Pediatrics (2012) Breastfeeding and the use of human milk. Pediatrics 129, e827-e841.

18. McInnes RJ \& Stone DH (1999) Promotion of exclusive breastfeeding. Lancet 354, 161-162.

19. Larque E, Sabater-Molina M \& Zamora S (2007) Biological significance of dietary polyamines. Nutrition 23, 87-95.

20. Buts JP, De Keyser N, Kolanowski J, et al. (1993) Maturation of villus and crypt cell functions in rat small intestine. Role of dietary polyamines. Dig Dis Sci 38, 1091-1098. 
21. Capano G, Bloch KJ, Carter EA, et al. (1998) Polyamines in human and rat milk influence intestinal cell growth in vitro. J Pediatr Gastroenterol Nutr 27, 281-286.

22. Deloyer P, Peulen O \& Dandrifosse G (2005) Intestinal effects of long-lasting spermine ingestion by suckling rats. Exp Physiol 90, 901-908.

23. Gao JH, Guo LJ, Huang ZY, et al. (2013) Roles of cellular polyamines in mucosal healing in the gastrointestinal tract.J Physiol Pharmacol 64, 681-693.

24. Harada E, Hashimoto Y \& Syuto B (1994) Orally administered spermine induces precocious intestinal maturation of macromolecular transport and disaccharidase development in suckling rats. Comp Biochem Physiol A Physiol 109, 667-673.

25. Kaouass M, Deloyer P, Wery I, et al. (1996) Analysis of structural and biochemical events occurring in the small intestine after dietary polyamine ingestion in suckling rats. Dig Dis Sci $\mathbf{4 1}$, 1434-1444.

26. van Wettere WH, Willson NL, Pain SJ, et al. (2016) Effect of oral polyamine supplementation pre-weaning on piglet growth and intestinal characteristics. Animal 10, 1655-1659.

27. Wang J, Li GR, Tan BE, et al. (2015) Oral administration of putrescine and proline during the suckling period improves epithelial restitution after early weaning in piglets. J Anim Sci 93, 1679-1688.

28. ter Steege JC, Buurman WA \& Forget PP (1997) Spermine induces maturation of the immature intestinal immune system in neonatal mice. J Pediatr Gastroenterol Nutr 25, 332-340.

29. Perez-Cano FJ, Gonzalez-Castro A, Castellote C, et al. (2010) Influence of breast milk polyamines on suckling rat immune system maturation. Dev Comp Immunol 34, 210-218.

30. Sanchez M, Suarez L, Andres MT, et al. (2017) Modulatory effect of intestinal polyamines and trace amines on the spontaneous phasic contractions of the isolated ileum and colon rings of mice. Food Nutr Res 61, 1321948.

31. Kim YC, Sim JH, Choi W, et al. (2008) Relaxant effect of spermidine on acetylcholine and high $\mathrm{k}$-induced gastric contractions of Guinea-pig. Korean J Physiol Pharmacol 12, 59-64.

32. Borowsky B, Adham N, Jones KA, et al. (2001) Trace amines: identification of a family of mammalian $G$ protein-coupled receptors. Proc Natl Acad Sci U S A 98, 8966-8971.

33. Gidrewicz DA \& Fenton TR (2014) A systematic review and meta-analysis of the nutrient content of preterm and term breast milk. BMC Pediatr 14, 216.

34. Plaza-Zamora J, Sabater-Molina M, Rodriguez-Palmero M, et al. (2013) Polyamines in human breast milk for preterm and term infants. Br J Nutr 110, 524-528.

35. Ali MA, Strandvik B, Palme-Kilander C, et al. (2013) Lower polyamine levels in breast milk of obese mothers compared to mothers with normal body weight. J Hum Nutr Diet 26 Suppl. 1, 164-170.

36. Atiya Ali M, Strandvik B, Sabel KG, et al. (2014) Polyamine levels in breast milk are associated with mothers' dietary intake and are higher in preterm than full-term human milk and formulas. J Hum Nutr Diet 27, 459-467.

37. Gomez-Gallego C, Kumar H, Garcia-Mantrana I, et al. (2017) Breast milk polyamines and microbiota interactions: impact of mode of delivery and geographical location. Ann Nutr Metab 70, 184-190.

38. Perez M, Ladero V, Redruello B, et al. (2016) Mastitis modifies the biogenic amines profile in human milk, with significant changes in the presence of histamine, putrescine and spermine. PLOS ONE 11, e0162426.

39. Loret S, Brolet P, Pierzynowski S, et al. (2000) Pancreatic exocrine secretions as a source of luminal polyamines in pigs. Exp Physiol 85, 301-308.
40. Ardeshir A, Narayan NR, Mendez-Lagares G, et al. (2014) Breast-fed and bottle-fed infant rhesus macaques develop distinct gut microbiotas and immune systems. Sci Transl Med 6, 252ra120.

41. Kitada Y, Muramatsu K, Toju H, et al. (2018) Bioactive polyamine production by a novel hybrid system comprising multiple indigenous gut bacterial strategies. Sci Adv $\mathbf{4}$, eaat0062.

42. Nakamura A, Ooga T \& Matsumoto M (2019) Intestinal luminal putrescine is produced by collective biosynthetic pathways of the commensal microbiome. Gut Microbes 10, 159-171.

43. Gomez-Gallego C, Collado MC, Ilo T, et al. (2012) Infant formula supplemented with polyamines alters the intestinal microbiota in neonatal BALB/cOlaHsd mice. J Nutr Biochem 23, 1508-1513.

44. Gomez-Gallego C, Collado MC, Perez G, et al. (2014) Resembling breast milk: influence of polyamine-supplemented formula on neonatal BALB/cOlaHsd mouse microbiota. $\mathrm{BrJ}$ Nutr 111, 1050-1058.

45. Gomez-Gallego C, Garcia Romo M, Frias R, et al. (2017) Mice exposed to infant formula enriched with polyamines: impact on host transcriptome and microbiome. Food Funct 8, $1622-1626$.

46. Milovic V (2001) Polyamines in the gut lumen: bioavailability and biodistribution. Eur $J$ Gastroenterol Hepatol $\mathbf{1 3}$, 1021-1025.

47. Escribano MI \& Legaz ME (1988) High performance liquid chromatography of the dansyl derivatives of putrescine, spermidine, and spermine. Plant Physiol 87, 519-522.

48. Velasco L, Secades L, Bordallo C, et al. (2008) Role of putrescine on androgen-elicited positive inotropism in the left atrium of rats. J Cardiovasc Pharmacol 52, 161-166.

49. Cohen J (editor) (1988) Statistical Power Analysis for the Behavioral Sciences, 2nd ed. Hillsdale, NJ: Lawrence Erlbaum Associates.

50. Pollack PF, Koldovsky O \& Nishioka K (1992) Polyamines in human and rat milk and in infant formulas. Am J Clin Nutr 56, 371-375.

51. Romain N, Dandrifosse G, Jeusette F, et al. (1992) Polyamine concentration in rat milk and food, human milk, and infant formulas. Pediatr Res 32, 58-63.

52. Dominguez-Bello MG, De Jesus-Laboy KM, Shen N, et al. (2016) Partial restoration of the microbiota of cesarean-born infants via vaginal microbial transfer. Nat Med 22, 250-253.

53. Urbaniak C, Angelini M, Gloor GB, et al. (2016) Human milk microbiota profiles in relation to birthing method, gestation and infant gender. Microbiome $\mathbf{4}, 1$.

54. Cabrera-Rubio R, Mira-Pascual L, Mira A, et al. (2016) Impact of mode of delivery on the milk microbiota composition of healthy women. J Dev Orig Health Dis 7, 54-60.

55. Kumar H, du Toit E, Kulkarni A, et al. (2016) Distinct patterns in human milk microbiota and fatty acid profiles across specific geographic locations. Front Microbiol 7, 1619.

56. Pannaraj PS, Li F, Cerini C, et al. (2017) Association between breast milk bacterial communities and establishment and development of the infant gut microbiome. JAMA Pediatr 171, 647-654.

57. Timmerman HM, Rutten N, Boekhorst J, et al. (2017) Intestinal colonisation patterns in breastfed and formula-fed infants during the first 12 weeks of life reveal sequential microbiota signatures. Sci Rep 7, 8327.

58. Buts JP (1992) Refractory diarrhea in infants: experimental basis of current therapeutic perspectives. Bull Mem Acad R Med Belg 147, 209-213; discussion 213-207.

59. Dorhout B, van Beusekom CM, Huisman M, et al. (1996) Estimation of 24-hour polyamine intake from mature human milk. J Pediatr Gastroenterol Nutr 23, 298-302. 
60. Spizzirri UG, Puoci F, Lemma F, et al. (2019) Biogenic amines profile and concentration in commercial milks for infants and young children. Food Addit Contam Part A Chem Anal Control Expo Risk Assess 36, 337-349.

61. Barbieri F, Montanari C, Gardini F, et al. (2019) Biogenic amine production by lactic acid bacteria: a review. Foods $\mathbf{8}, 17$.

62. Meana C, Rubin JM, Bordallo C, et al. (2016) Correlation between endogenous polyamines in human cardiac tissues and clinical parameters in patients with heart failure. $J$ Cell Mol Med 20, 302-312.

63. Lambertos A, Ramos-Molina B, Cerezo D, et al. (2018) The mouse Gm853 gene encodes a novel enzyme: Leucine decarboxylase. Biochim Biophys Acta Gen Subj 1862, 365-376.

64. Sutton CR \& King HK (1962) Inhibition of leucine decarboxylase by thiol-binding reagents. Arch Biochem Biophys 96, 360-370

65. Bast E, Hartmann T \& Steiner M (1971) Studies on a valine carboxy-lyase of Bacillus sphaericus. Arch Mikrobiol 79, 12-24.

66. Coton M, Delbes-Paus C, Irlinger F, et al. (2012) Diversity and assessment of potential risk factors of Gram-negative isolates associated with French cheeses. Food Microbiol 29, 88-98.

67. Delbes-Paus C, Pochet S, Helinck S, et al. (2012) Impact of Gram-negative bacteria in interaction with a complex microbial consortium on biogenic amine content and sensory characteristics of an uncooked pressed cheese. Food Microbiol 30, 74-82.

68. Soto A, Martin V, Jimenez E, et al. (2014) Lactobacilli and bifidobacteria in human breast milk: influence of antibiotherapy and other host and clinical factors. $J$ Pediatr Gastroenterol Nutr 59, 78-88.

69. Sharma C, Singh BP, Thakur N, et al. (2017) Antibacterial effects of Lactobacillus isolates of curd and human milk origin against food-borne and human pathogens. 3 Biotech 7, 31.

70. Jost T, Lacroix C, Braegger C, et al. (2013) Assessment of bacterial diversity in breast milk using culture-dependent and culture-independent approaches. Br J Nutr 110, 1253-1262.

71. Akagawa S, Tsuji S, Onuma C, et al. (2019) Effect of delivery mode and nutrition on gut microbiota in neonates. Ann Nutr Metab 74, 132-139.

72. Borewicz K, Gu F, Saccenti E, et al. (2019) Correlating infant faecal microbiota composition and human milk oligosaccharide consumption by microbiota of one-month old breastfed infants. Mol Nutr Food Res 24, e1801214.

73. Pugin B, Barcik W, Westermann P, et al. (2017) A wide diversity of bacteria from the human gut produces and degrades biogenic amines. Microb Ecol Health Dis 28, 1353881.
74. Madan JC, Farzan SF, Hibberd PL, et al. (2012) Normal neonatal microbiome variation in relation to environmental factors, infection and allergy. Curr Opin Pediatr 24, 753-759.

75. Boix-Amoros A, Collado MC \& Mira A (2016) Relationship between milk microbiota, bacterial load, macronutrients, and human cells during lactation. Front Microbiol 7, 492.

76. Cabrera-Rubio R, Collado MC, Laitinen K, et al. (2012) The human milk microbiome changes over lactation and is shaped by maternal weight and mode of delivery. Am J Clin Nutr $\mathbf{9 6}$, 544-551.

77. Palmer C, Bik EM, DiGiulio DB, et al. (2007) Development of the human infant intestinal microbiota. PLoS Biol 5, e177.

78. Penders J, Vink C, Driessen C, et al. (2005) Quantification of Bifidobacterium spp., Escherichia coli and Clostridium difficile in faecal samples of breast-fed and formula-fed infants by real-time PCR. FEMS Microbiol Lett 243, 141-147.

79. Ladero V, Fernandez M, Calles-Enriquez M, et al. (2012) Is the production of the biogenic amines tyramine and putrescine a species-level trait in enterococci? Food Microbiol 30, 132-138.

80. Ladero V, Cañedo E, Pérez M, et al. (2012) Multiplex qPCR for the detection and quantification of putrescine-producing lactic acid bacteria in dairy products. Food Control 27, 307-313.

81. Campeotto F, Butel MJ, Kalach N, et al. (2004) High faecal calprotectin concentrations in newborn infants. Arch Dis Child Fetal Neonatal Ed 89, F353-355.

82. Sabater-Molina M, Larque E, Torrella F, et al. (2009) Effects of dietary polyamines at physiologic doses in early-weaned piglets. Nutrition 25, 940-946.

83. Tang L, Cheng CY, Sun X, et al. (2016) The extracellular Calcium-sensing receptor in the intestine: evidence for regulation of colonic absorption, secretion, motility, and immunity. Front Physiol 7, 245.

84. Nicholson JK, Holmes E, Kinross J, et al. (2012) Host-gut microbiota metabolic interactions. Science 336, 1262-1267.

85. Pegg AE (2013) Toxicity of polyamines and their metabolic products. Chem Res Toxicol 26, 1782-1800.

86. Sharmin S, Sakata K, Kashiwagi K, et al. (2001) Polyamine cytotoxicity in the presence of bovine serum amine oxidase. Biochem Biophys Res Commun 282, 228-235.

87. Linares DM, del Rio B, Redruello B, et al. (2016) Comparative analysis of the in vitro cytotoxicity of the dietary biogenic amines tyramine and histamine. Food Chem 197, 658-663.

88. Del Rio B, Redruello B, Linares DM, et al. (2019) The biogenic amines putrescine and cadaverine show in vitro cytotoxicity at concentrations that can be found in foods. Sci Rep $\mathbf{9}, 120$. 